\title{
Hydatid Cyst of Submandibular Region
}

\author{
https://doi.org/10.47210/bjohns.2020.v28i3.279
}

Jyotirmoy Phookan, ${ }^{1}$ Pritam Chatterjee, ${ }^{2}$ Sritama De ${ }^{3}$

$\underline{\text { Introduction }}$

ABSTRACT

Hydatid cysts in the neck are relatively exceptional. We report a rare case of a hydatid cyst localized in the right submandibular region of the neck with a second cyst in lung discussing diagnostic and management algorithm with brief review of literature. Case Report

A 6 years old boy presented to us in Gauhati Medical College \& Hospital with a gradually enlarging painless swelling in right submandibular region since last 4 months. The patient was thoroughly evaluated. Imaging and FNAC was performed. Suspected common locations were also screened prior to surgery. He was treated with total pericystectomy and followed up regularly.

$\underline{\text { Discussion }}$

Hydatid disease is a widespread public health problem in developing countries. The possibility of hydatid disease, especially in endemic regions, may always be considered in the differential diagnosis of mesenchymal neoplasms or soft tissue cystic masses in the neck. Radiologic imaging modalities in such cases are mandatory for the diagnosis. The prognosis is excellent in hydatid cyst cases treated with total removal of the cyst without rupture.

$\underline{\text { Keywords }}$

Hydatid Cyst; Submandibular

$\mathrm{H}$ ydatid cyst is an infectious disease which is most commonly caused by Echinococcus granulosus and less commonly by Echinococcus multilocularis. Humans are accidental intermediate hosts in echinococcus lifecycle, as infection of human beings represents a terminal event. Dogs are the main host, and animals like cattle, sheep, horse and pig are intermediate hosts in the disease. Parasite eggs that penetrate the organism hatch in small intestine of the main host, pass into portal venous system or lymphatic system and reach liver and lungs, and finally form hydatid cyst lesions.

1 - Department of Otorhinolaryngology \& Head-Neck Surgery, Assam Medical College \& Hospital, Dibrugarh, Assam

2 - Department of Otorhinolaryngology \& Head-Neck Surgery, Bankura Sammilani Medical College \& Hospital, Bankura

3 - Department of Otorhinolaryngology \& Head-Neck

Surgery, Jag Pravesh Chandra Hospital, New Delhi

\section{Corresponding author:}

Dr Jyotirmoy Phookan

email: jpphookan@hotmail.com
Moreover, they can cross hepatic sinusoid or pulmonary capillary barriers, and embryos get into systemic circulation and can settle in all the organs and structures in the body. ${ }^{1,4}$ Typically, a man is exposed to the organism by ingestion of contaminated vegetable or meat.

Infection may also occur when playing with dogs harbouring tapeworm, as eggs cling to their fur. The larval form may invade any organ system, and distribution of infection is limited only by blood flow and filtration. Hydatid cyst is most frequently involved in liver and lungs, and rarely in the bone, brain, eye, heart, kidney, spleen and parotid gland. 3,5 Atypical localization of hydatid cyst may be challenged the diagnosis of hydatid disease. ${ }^{2}$ The neck is one of the atypical sites of hydatid disease, accounting for no more than $1 \%$ of all hydatid sites. ${ }^{1,6}$

\section{Case report}

A 6 years old boy presented at Gauhati Medical College with a painless swelling in right submandibular region which he noticed 4 months back. The swelling had gradually increased in size however there was no history 


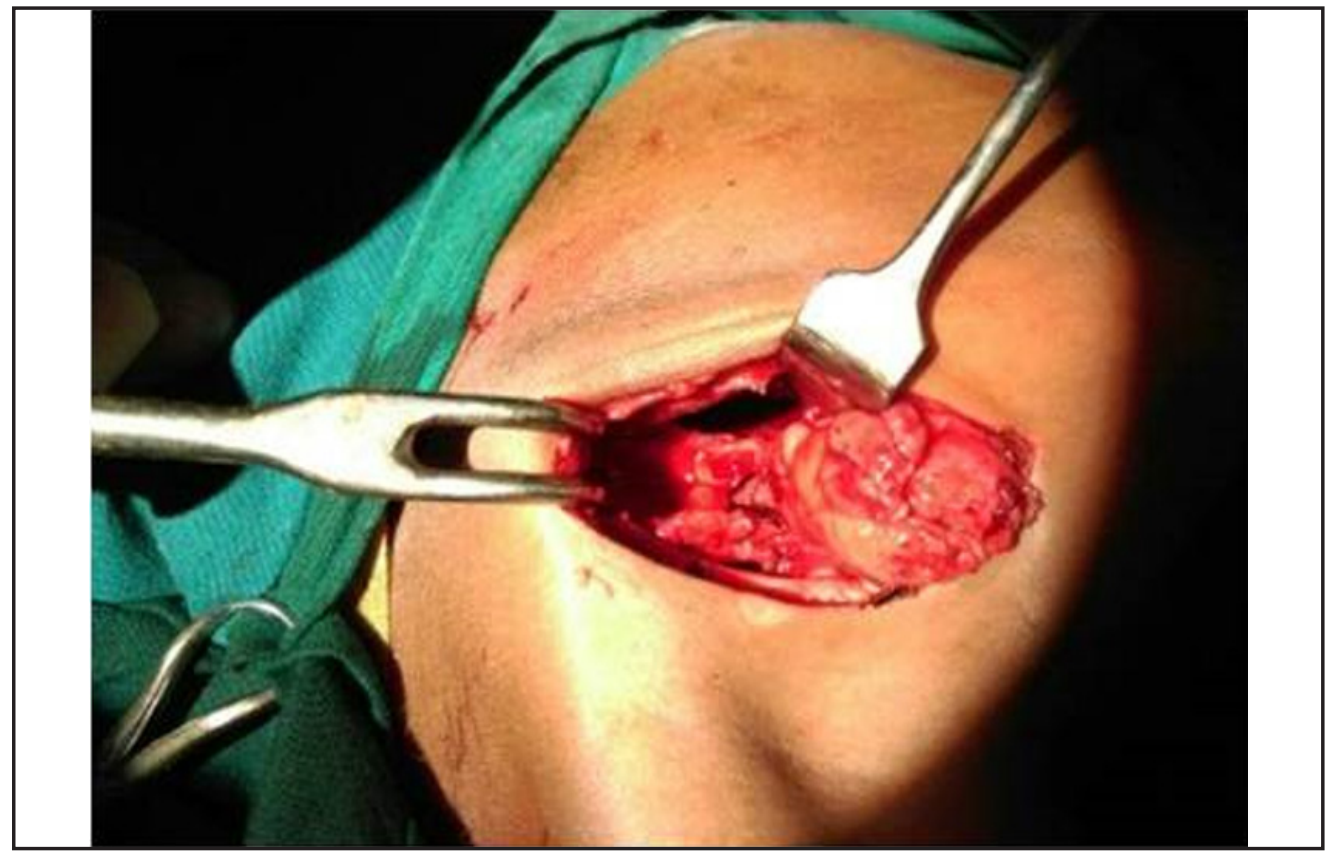

Fig. 1. Post-operative surgical site showing the submandibular region with intact submandibular gland.

(Approach through horizontal incision $1 \mathrm{~cm}$ below and along lower border of mandible, surgical step similar till exposure of submandibular triangle and gland, Cyst found separate from Submandibular gland, Submandibular gland transposed anteriorly, Peri cystectomy done preserving the Submandibular gland)

of increase in size while consuming food, fever difficulty in swallowing or any other systemic complains. On physical examination, there was a smooth, soft, cystic, non-tender, immobile mass of $4 \times 3 \mathrm{~cm}$ in right submandibular region. CECT of neck revealed a cystic lesion in pre-styloidcompartment of right parapharyngeal space, superiorly extending to base of skull, displacing submandibular gland inferiorly, medially protruding into nasopharyngeal and oropharyngeal air column, laterally compressing masticator space and displacing carotid sheath posteriorly. Suspecting a possible hydatid cyst, we also conducted a thorough imaging studies of more common locations for hydatid cyst which included USG whole abdomen, CECT of brain and HRCT of lungs. HRCT thorax revealed a well-defined cystic lesion of $68 \times 53 \mathrm{~mm}$ was noted in left lung predominantly involving upper lobe abutting mediastinal pleura around hilum and lateral costal pleura.

FNAC was only suggestive of a cystic lesion. In collaboration with CTVS department excision of neck \& lung cyst was done. Neck cyst was approached through an approximately $3 \mathrm{~cm}$ long horizontal incision
$1 \mathrm{~cm}$ below \& along lower border of mandible, and identified lying in right digastric triangle free from any significant attachment to nearby structures including the submandibular gland. The Submandibular gland was transposed anteriorly and total pericystectomy was

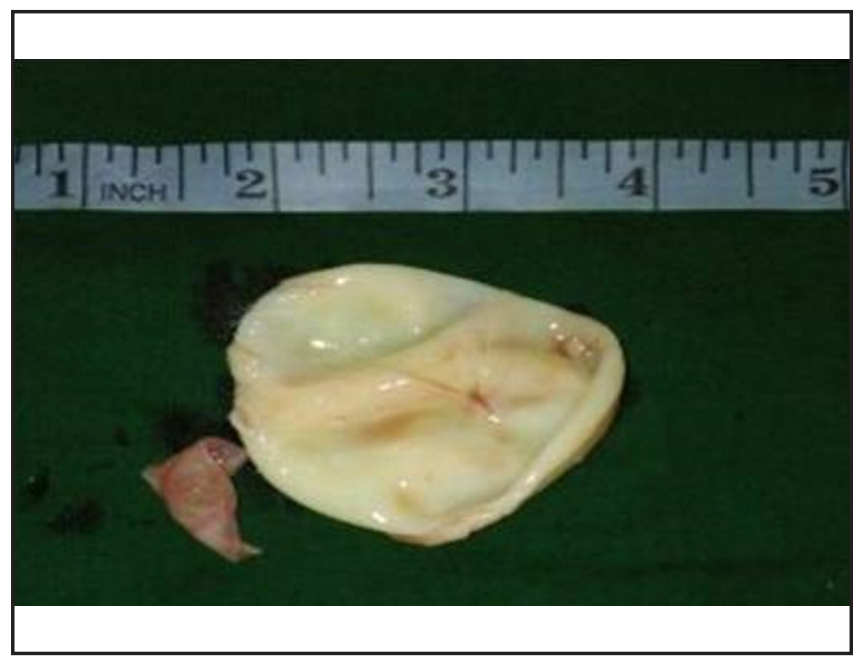

Fig 2. Post-operative specimen of the resected cyst and its germinative membrane. 


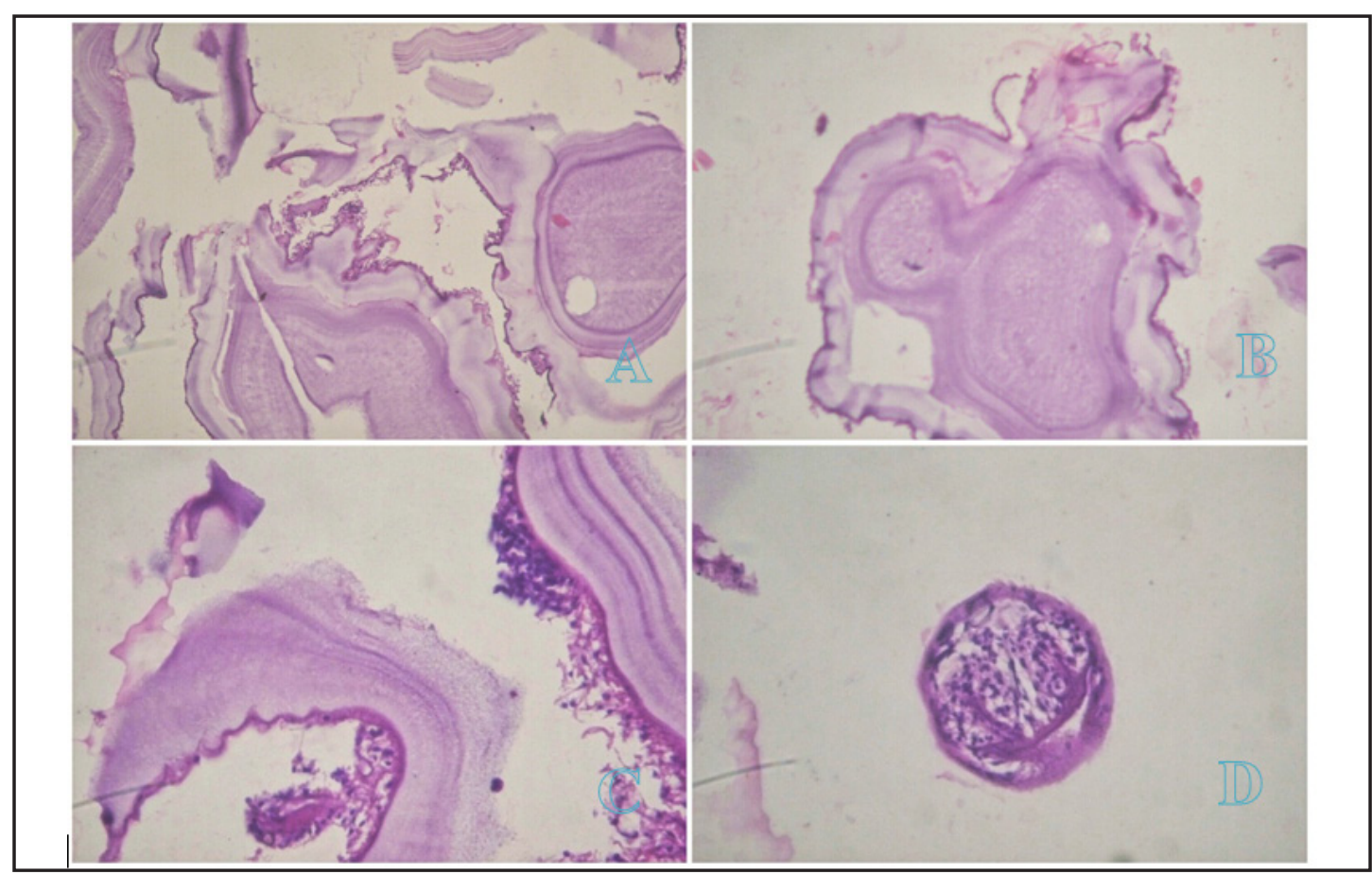

Fig. 3. Microscopic examination using Haematoxylin \& Eosin staining $(A=200 X, B=200 X, C=400 X, D=$ $600 \mathrm{X})$. (A, B, C) Microscopic examination of the specimen demonstrating a thick collagenous fibrotic cyst wall (D) free scolex in the cyst lumen.

performed preserving the submandibular gland. (Fig.

1) Grossly, it was a cystic mass with an outer smooth greyish white glistening surface measuring about $6 \times 4 \times 3 \mathrm{~cm}$. (Fig. 2) HPE showed cyst wall being lined by a germinative layer and composed of pale eosinophilic, acellular, laminated chitinous material suggestive of hydatid cyst. (Fig. 3) The lung cyst was excised subtotally due to significant air leakage form left upper lobe and closed with a chest drain. Rest of the hospital stay was uneventful. The patient is under yearly follow-up for the last 5 years without any local recurrence.

\section{Discussion}

Hydatid cysts at unusual sites have been reported all around the world. Hydatid disease in the neck is quite rare and the diagnosis is remarkably difficult because of presence of simulating and more common cystic pathological conditions in the neck.

Hydatid cyst is a diagnosis rarely considered, even in endemic zones, in the presence of a neck cyst. As such isolated cysts are more suggestive of congenital cyst or benign tumour. It displays the characteristic symptoms of benign tumour that grows slowly. It may also imitate cysts, abscess, hematoma or pseudocysts. Although the disease is generally asymptomatic, it may exhibit clinical symptoms depending on the size and location of the cyst and the pressure of the growing cyst. Physical examination may also reveal signs of laryngotracheal compression or even local infection following puncture or rupture of the cyst.

Medical imaging provides a considerable contribution, not only to confirm the diagnosis, but also to eliminate any other coexisting hydatid cysts, particularly in the liver, lungs and spleen. Ultrasound constitutes the first-line examination as it can visualise features highly suggestive of hydatid cyst (detachment of the membrane, multilocular appearance and peripheral calcifications), similar to those observed in other visceral sites, particularly the liver, corresponding to Gharbi's classification. ${ }^{7}$ In the absence of these signs, differential diagnosis can include cystic lymphangioma, 
cold abscess, chronic haematoma, and epidermal cyst. The sensitivity and specificity of ultrasound have been estimated to be $90-95 \%{ }^{8}$

Positive hydatid serology confirms definitive diagnosis, while eosinophilia is nonspecific, as it is observed in any form of parasitic infestation. ${ }^{8}$ Serological tests are broadly used to substantiate the diagnosis. However, because of chances of false positives and negatives, imaging modalities remain more sensitive than serodiagnosis, especially with unusual sites. However, in our patient, ultrasound report did not diagnose hydatid cyst, probably the unusual site being the reason behind the miss. Abdomino-pelvic ultrasound and chest X-ray, should be performed in context to detect other hydatid sites in such cases. CT can be used to confirm suspected lesions.

Fine needle aspiration cytology can suggest the diagnosis by showing clear fluid characteristic of hydatid disease. ${ }^{8}$ However, this examination is considered to be dangerous due to risks of fissure, dissemination, anaphylactic reactions \& infectious inoculation. ${ }^{8}$

The definitive diagnosis is based on histology with direct microscopic examination demonstrating debris of lamellar membrane, hooklets or whole protoscolex during percutaneous aspiration, biopsy or examination of the resection specimen. This direct examination can determine the viability of the protoscolices.

Treatment of hydatid cyst of the neck is exclusively surgical. ${ }^{8}$ Total pericystectomy, removing the entire cyst, is the method of choice. ${ }^{8}$ Subtotal pericystectomy or resection of roof of the cyst are reserved to cases in which cyst arises in contact with nerves and vessels. ${ }^{8}$

Prior to incision, the surgical field must be protected by scolicidal solution to avoid any dissemination of scolices in event of accidental opening of the cyst. 8 Care must be taken while dissection for intact cyst delivery as the cyst fluid is highly anaphylactogenic and required medications for anaphylaxis management must be checked prior surgery. Accidental spillage is to be cleared immediately and surgical field may be guarded by sponges.

Injecting a scolicidal agent into the unopened cyst and walling off the operative field with sponges soaked in a scolicidal agent are the two most commonly employed scolicidal measures. ${ }^{9} 0.4 \%$ chlorhexidine gluconate, $1.5 \%$ cetrimide $-0.15 \%$ chlorhexidine $(10 \%$ Savlon ${ }^{\circledR}$ ), Povidone-iodine (Betadine ${ }^{\circledR}$ ) and $3 \%$ hydrogen peroxide are fastest acting effective scolicidal agents in their undiluted to half diluted forms in 5- and 10-minute exposure time on field and in 15 minutes on surgical sponges. ${ }^{9,10} \quad 0.24 \%$ sodium hypochlorite, $95 \%$ ethyl alcohol and 20\% saline are only effective scolicidal agents in undiluted form reaching maximum efficacy after 20 minutes..$^{9,10}$ All agents are to used cautiously as every one agent is locally and systemically toxic. Savlon appears to most widely available fastest acting least toxic scolicidal agent. There is no maximum volume guide. As these agents acts on the surface applied, effects \& toxicities are time, volume and area of application dependent.

Regular postoperative and long-term surveillance is required, essentially based on ultrasound, primarily to monitor appearance of residual cavity. Hydatid serology is performed six months and one year after surgery. Computed tomography is reserved for cases of suspected recurrence and surveillance of large residual cavities.

Medical treatment with imidazole derivatives (mebendazole and albendazole) has a limited place in treatment of hydatid disease of neck, as, when used alone, it only decreases size of the cysts and cyst sterilization is much longer to achieve. ${ }^{8}$ It is prescribed as an adjuvant to surgery, especially following intraoperative cyst rupture, postoperative recurrence or in cases of multiple hydatid cysts. ${ }^{8}$

Radiology in form of ultrasound appears to be the most reliable modality for surveillance after exclusive medical treatment by demonstrating reduction of size of cysts and/or increased intracystic density or echogenicity. Physical examination and serology contribute poorly, as hydatid cyst, even when dead, can remain antigenically active and can continue to stimulate host's immune system.

Hydatid disease is a widespread public health problem in developing countries. The possibility of hydatid disease, especially in endemic regions, may always be considered in the differential diagnosis of mesenchymal neoplasms or soft tissue cystic masses in 
the neck. Possibility of multiple lesion should always be kept in mind and if suspected, commoner locations must always be screened prior to surgery. Radiologic imaging modalities in such cases are mandatory for the diagnosis and post-operative follow-ups. The prognosis is excellent in hydatid cyst cases treated with total removal of the cyst without rupture. In case of intraoperative rupture or planned partial cystectomy is performed, the surgical field must be washed thoroughly by scolicidal solution to prevent recurrence and followed up regularly for early identification of possible recurrence.

\section{References}

1. Celik A, Turanli M, Kutun S, Delibasi T, Mengi N, Comert E, Aslan S, et al. Unusual location of hydatid cyst: soft tissue mass in the neck. Eur Arch Otorhinolaryngol 2006; 263(12):11471150 .

2. Ahmad S, Jalil S, Saleem Y, Suleman BA, Chughtai N. Hydatid cysts at unusual sites: reports of two cases in the neck and breast. J Pak Med Assoc 2010;60(3):232-234.

3. Sogut O, Ozgonul A, Bitiren M, Kose R, Cece H. Primary hydatid cyst in the deltoid muscle: an unusual localization. Int $\mathrm{J}$
Infect Dis 2010;14(3): 347-348.

4. Unal AE, Ulukent SC, Bayar S, Demirkan A, Akgul H. Primary hydatid cyst of the axillary region: report of a case. Surg Today 2001;31(9):803-805.

5. Darabi M, Varedi P, Mohebi AR, Mahmoodi S, Nabavizadeh SA, Erfan A, Ostadali Makhmalbaf A, et al. Hydatid cyst of the parotid gland. Oral Maxillofac Surg 2009; 13(1):33-35.

6. Dirican A, Unal B, Kayaalp C, Kirimlioglu V. Subcutaneous hydatid cysts occurring in the palm and the thigh: two case reports. J Med Case Reports 2008; 2:273.

7. Gharbi HA, Hassini W, Brauner MO, et al. Ultrasound examination of the hydatic liver. Radiology 1981; 139:45963.

8. Hmidi M, Touiheme N, Rbai M, Messary A. Isolated hydatid cyst of the neck: An unusual site. European Annals of Otorhinolaryngology, Head and Neck diseases. 2012;129:108110

9. Besim H, Karayalcin K, Hamamci O, Gongor C, Korkmaz C. Scolicidal Agents in Hydatid Cyst Surgery. HPB Surgery 1998; 10:347-51

10. Borazan E, Gökalp MA, Zer Y, Aksoy N, Aytekin A, Yilmaz L. Time-related comparison of scolicidal activity of the different substances used in Hydatid Cyst. East J Med. 2019; 24(4): 45762 . 